\title{
PREVALÊNCIA DE ATRASO DO DESENVOLVIMENTO NEUROPSICOMOTOR EM PRÉ-ESCOLARES
}

\author{
PREVALENCE OF NEUROPSYCHOMOTOR \\ DEVELOPMENT DELAY IN PRESCHOOL CHILDREN
}

\author{
Jamili Anbar Torquato ${ }^{1}$ \\ Janine Batista Paes ${ }^{1}$ \\ Maria Cristiane C. Bento ${ }^{1}$ \\ Glaucy Mara P. N. Saikai ${ }^{1}$ \\ Juliana Novaes Souto ${ }^{1}$ \\ Elbimeire de Almeida M. Lima ${ }^{1}$ \\ Luiz Carlos de Abreu ${ }^{2}$
}

Torquato JA et al. Prevalência de atraso do desenvolvimento neuropsicomotor em préescolares. Rev Bras Cresc e Desenv Hum 2011; 21(2): 259-268.

\section{RESUMO}

O desenvolvimento infantil é um processo de mudanças relacionado com a idade e influenciado pelo ambiente em que a criança vive. Desta maneira, o objetivo é verificar o a prevalência de atraso do desenvolvimento neuropsicomotor (DNPM) em pré-escolares. Procedimentos: tratou-se de um estudo transversal analítico realizado em três abrigos e uma escola particular da Zona Leste de São Paulo-SP, na qual participaram 81 crianças. Aplicou-se um questionário aos responsáveis pelas crianças participantes e para avaliar o desenvolvimento neuropsicomotor das crianças foi utilizado o Teste Denver II. Os resultados revelaram a prevalência de atraso no DNPM em crianças institucionalizadas $(31,6 \%), p=0,06$. A linguagem foi a área mais freqüentemente afetada nas crianças com suspeita de atraso. Em relação ao suporte familiar e condições ambientais não houve diferenças estatisticamente significantes nas crianças institucionalizadas. Verificou-se que os resultados não significativos talvez sejam conseqüência da relação de apego que as crianças institucionalizadas adquirem entre si e da convivência com um maior número de crianças. Conclusão: o presente estudo não encontrou diferenças estatisticamente significativas no desenvolvimento neuropsicomotor de crianças institucionalizadas e não-institucionalizadas, porém houve maior prevalência de suspeita de atraso nas crianças institucionalizadas.

Palavras-chave: desenvolvimento infantil; criança institucionalizada; reabilitação; crianças.

1 Departamento de Ciências da Saúde da Universidade Cruzeiro do Sul - UNICSUL. E-mail: jamilianbar@yahoo.com. Universidade Cruzeiro do Sul - UNICSUL. Rua Taiuvinha, 31.São Paulo,SP. Departamento: Centro de Ciência Biológicas da Saúde - UNICSUL.

2 Laboratório de Escrita Científica. Faculdade de Medicina do ABC. Santo André, SP. e-mail: abreu.luizcarlos@gmail.com Correspondência para: Jamili Anbar Torquato. Rua Consolação, 3563 ,122 - CEP: 01416-001. São Paulo - SP. Cel: (11) 98188839. E-mail: jamilianbar@yahoo.com. Agência de fomento e auxílio: Programa Institucional de Bolsas de Iniciação Científica (PIBIC) - UNICSUL 


\begin{abstract}
The child development is a process of age-related changes and influenced by the environment where the child lives. This way, the goal is to determine the prevalence of delay development neuropsychomotor in preschool children. Procedures: this was an analytical cross-sectional study performed in three shelters and a private school in the East zone of São Paulo-SP, attended 81 children. A questionnaire was applied to children participating and to evaluate the development neuropsychomotor of children was used testing Denver II. The results revealed the prevalence of late DNPM institutionalised children $(31.6 \%), p=0.06$. The language was the area most often affected in children with suspected delays. In relation to the support of family and environmental conditions there were statistically significant differences in institutionalised children. The insignificant results may be a consequence of the relationship of attachment that institutionalized children acquire each other and coexistence with a greater number of children. Conclusion: this study found no statistically significant differences in the development of institutionalized children neuropsicomotor and non-institutionalized, but there were higher prevalence of suspected delay in institutionalised children.
\end{abstract}

key words: child development; institutionalised children; rehabilitation children.

\section{INTRODUÇÃO}

Com o crescimento ocorre a aquisição gradual das habilidades latentes de uma criança. Estágio por estágio, as primeiras aquisições motoras são modificadas, elaboradas e adaptadas para padrões e habilidades de movimentos mais finos e seletivos ${ }^{1}$, sendo este desenvolvimento um processo de mudança no comportamento motor, o qual está relacionado com a idade da criança ${ }^{2}$.

Uma grande preocupação se concentra no fato de que há maior vulnerabilidade biológica em lactentes que tiveram condições desfavoráveis ao nascimento e continuam sofrendo adversidades ao longo do primeiro ano de vida, quando ocorrem importantes mudanças em seu sistema nervoso central ${ }^{3}$. O sistema nervoso da criança está em constante transformação e a combinação entre os fatores genéticos e ambientais é o que determina o seu desenvolvimento ${ }^{4}$.

A interação entre o ambiente e individuo é enfatizada pela teoria ecológica proposta por James Gibson, no qual o indivíduo explora ati- vamente o ambiente. Desta forma, o contexto ou ambiente em que as crianças estão inseridas e as exigências das tarefas propostas têm grande influência na aquisição de novas habilidades $^{5,6}$.

A instituição de abrigo consiste em um ambiente ecológico de extrema importância para as crianças e adolescentes institucionalizados. É o local onde realizam um grande número de atividades, funções e interações, sendo um ambiente com potencial para o desenvolvimento de relações recíprocas de equilíbrio, de poder e de afeto. ${ }^{7}$ Ainda assim, essas crianças sofrem privação materna associada à carência de estímulos podendo acarretar em atraso no desenvolvimento motor. ${ }^{8} \mathrm{~A}$ família proporciona para a criança um ambiente de carinho, amor, segurança, proteção, e também valores , religião, alem de condições materiais, lazer, passeios, existe o apoio emocional, todo este suporte proporciona a criança um desenvolvimento saudável.

É importante a detecção precoce de alterações no desenvolvimento motor da criança para que a intervenção possa ocorrer nos perí- 
odos oportunos para o desenvolvimento das funções visuais e motoras. ${ }^{9}$

Desta maneira, o objetivo é verificar a prevalência de atraso do desenvolvimento neuropsicomotor (DNPM) em pré-escolares.

\section{MÉTODO}

Tratou-se de um estudo transversal analítico realizado na Zona Leste de São PauloSP ,a amostra foi estabelecida através de escolha de instituições com crianças dentro dos critérios de inclusão do trabalho e que fosse permitido realizar a pesquisa. Participaram 81 crianças em fase pré-escolar, ambos os sexos, sendo que 38 eram institucionalizadas há mais de 6 meses (em três abrigos) e freqüentavam escola pública em período integral ou meio período e 43 crianças não-institucionalizadas que freqüentavam escola particular por período integral.

Os critérios de inclusão foram ser institucionalizadas há mais de 6 meses, ter até 6 anos, ou freqüentar escola particular, foram exclusos do estudo crianças que realizaram cirurgia ortopédica recente, que apresentaram patologias neurológicas ou algum déficit auditivo/cognitivo, conforme constava no prontuário ou relato do responsável.

Para registrar informações sobre o histórico da criança foi utilizado um questionário elaborado a partir do modelo que Filipini ${ }^{10}$ aplicou em sua pesquisa, porém com acréscimo de variáveis relacionadas aos estímulos dos cuidadores e ao suporte familiar. O questionário aplicado para as crianças institucionalizadas foi modificado e adaptado em relação ao das crianças não institucionalizadas, pois havia questões em relação à família, que não seriam respondidas, por ter muitas crianças abandonadas, ou sem visita dos pais. Os questionários foram respondidos após a assinatura do Termo de Consentimento Livre e Esclarecidos assinado pelos responsáveis.
Para identificar a criança com suspeita de atraso no DNPM, foi utilizado The Denver Developmental Screening Test II (Denver II), elaborado e revisado por Frankenburg ${ }^{11}$, considerado adequado para avaliar o crescimento e desenvolvimento da criança de 0 a 6 anos do ponto de vista neuropsicomotor.

O Denver II possui 125 itens organizados em forma de teste e divididos em quatro aspectos: pessoal-social (aspectos da socialização da criança dentro e fora do ambiente familiar), adaptação motora fina (coordenação e manipulação de pequenos objetos), linguagem (produção de som, compreensão e uso da linguagem) e motricidade ampla (controle motor corporal, sentar, caminhar, pular).

Foram considerados casos indicativos de suspeita de atraso no desenvolvimento aqueles em que a criança apresentou dois ou mais itens de atenção e/ou dois ou mais itens de falha ${ }^{11}$.

Antes da realização do teste de Denver II, as pesquisadoras foram treinadas com base na metodologia previamente estabelecida no respectivo manual de treinamento e foi feita uma ambientação com as crianças por meio de brincadeiras e jogos, diminuindo assim a possibilidade de erros de classificação devido a possíveis alterações no comportamento das crianças.

A variável de desfecho suspeita de atraso foi tratada como variável dicotômica (Denver II, suspeito de atraso versus sem suspeita de atraso) e a amostra foi dividida em 2 grupos: grupo 1, na qual pertenciam as crianças institucionalizadas, e grupo 2 , referente às crianças não institucionalizadas. Para análise dos dados foi utilizado o pacote estatístico Statistical Package for the Social Sciences (SPSS), Windows, versão 13.0, sendo realizada uma análise descritiva das variáveis qualitativas por meio de freqüências relativa e absoluta; e para as variáveis quantitativas, medidas de dispersão e de tendência central. $\mathrm{Na}$ análise bivariada foi utilizado o teste qui- 
quadrado e para comparação de médias o teste $\mathrm{t}$ de Student. Admitiu-se um á=0,05.

Este estudo foi aprovado pelo Comitê de Ética em Pesquisa na Universidade Cruzeiro do Sul, estando de acordo com as normas vigentes na Resolução Nº196/96 do Conselho Nacional de Saúde/Ministério da Saúde, e suas complementares.

\section{RESULTADOS}

Das 81 crianças que participaram do estudo, $51,9 \%(\mathrm{n}=42)$ eram do sexo feminino e $48,1 \%(n=39)$ do sexo masculino. A população estudada foi dividida em dois grupos: grupo 1 com 38 crianças (46,9\%) que pertenciam a três instituições de abrigos; e grupo 2 com 43 crianças $(53,1 \%)$ que freqüentavam uma escola particular em período integral. A idade das crianças de ambos os grupos variou entre 17 e 82 meses, no grupo 1 com uma média de $50,71 \pm 17,81$ meses e no grupo 2 foi de 45,81 $\pm 17,63$ meses.

Verificou-se que todas as crianças apresentaram espaço adequado para brincar, sendo que os locais considerados para brincar foram: quintal, quarto, sala de estar e/ou sala apropriada. As crianças do grupo 1 tinham dois ou mais dos locais citados para brincar e $41,8 \%(n=18)$ dos responsáveis do grupo 2 responderam que as crianças tinham a oportunidade de brincar em dois locais citados, sendo que $58,2 \%(n=25)$ das crianças brincavam em apenas um dos locais citados.

Todos os responsáveis relataram que as crianças possuíam brinquedos adequados para a idade e alimentação regular e apropriada. Dessa forma pode-se considerar que as condições ambientais mostraram-se adequadas em ambos os grupos.

No grupo 1, a média foi de 22,82 crianças residentes nos três abrigos e o tempo mínimo de institucionalização foi de 7 meses, sendo este um período propício para que as crianças já estivessem adaptadas ao ambiente do abrigo. $\mathrm{Na}$ instituição, essas crianças conviviam com uma média de 22,42 crianças (variando entre 5 e 25 crianças), sendo aproximadamente 2 funcionários responsáveis por 10 crianças.

No grupo 2, o tempo que as crianças freqüentavam a escola particular em período integral variou entre 6 e 54 meses. Em relação à convivência com outras crianças, a média foi de 17,30 crianças durante o período escolar, sendo um funcionário responsável por cerca de 20 crianças. Esses dados estão representados detalhadamente na tabela 1 .

Tabela 1: Caracterização de pré-escolares em abrigos ou escola particular da Zona Leste de São Paulo.

\begin{tabular}{lcc}
\hline & \multicolumn{2}{c}{ Crianças } \\
\hline \multirow{2}{*}{ Variáveis } & Não institucionalizadas & Institucionalizadas \\
\hline Número de crianças com que convive & Média $( \pm \mathrm{DP})$ & Média $( \pm \mathrm{DP})$ \\
Número de funcionários responsáveis & $17,30 \pm 7,03$ & $22,42 \pm 5,27$ \\
& $1,0 \pm 0,0$ & $1,92 \pm 0,27$ \\
\hline
\end{tabular}

A prevalência da suspeita de atraso no DNPM foi de $31,6 \%$ no grupo 1 e de $14 \%$ no grupo 2. Não houve atraso significante no DNPM nas crianças institucionalizadas e não institucionalizadas, $\mathrm{p}=0,067$ (Tabela 2). No grupo 1, o setor que houve maior prevalência de falhas e/ou atenção quando aplicado o teste de Denver II foi o da linguagem, e no grupo 2, foram os setores pessoal-social e linguagem (Tabela 3). 
Tabela 2: Desenvolvimento neuropsicomotor de pré escolares em abrigos ou escola particular da Zona Leste de São Paulo.

\begin{tabular}{lccc}
\hline \multicolumn{3}{c}{ Desenvolvimento Neuropsicomotor } \\
\hline Criança & Sim & Suspeita de atraso & Total \\
& $\mathrm{n}(\%)$ & $\mathrm{Não}$ & $\mathrm{n}(\%)$ \\
\hline Institucionalizada & $12(66,6)$ & $26(41,3)$ & 38 \\
Não Institucionalizada & $6(33,3)$ & $37(58,7)$ & 43 \\
Total & $18(100)$ & $63(100)$ & 81 \\
\hline
\end{tabular}

* $\mathrm{p}=0,067$

Tabela 3: Prevalência de suspeita de atraso no DNPM em relação cada domínio avaliado pelo teste de Denver II nos pré escolares de abrigos e de escola particular da Zona Leste de São Paulo.

\begin{tabular}{lrrrrrrrr}
\hline & \multicolumn{7}{c}{ Desenvolvimento Neuropsicomotor (Teste de Denver II) } \\
\hline \multirow{2}{*}{ Tipo de criança } & \multicolumn{2}{c}{ Pessoal-Social } & \multicolumn{2}{c}{ Motor-fino } & \multicolumn{2}{c}{ Linguagem } & \multicolumn{2}{c}{ Motor-grosso } \\
& $\mathrm{N}$ & $\%$ & $\mathrm{n}$ & $\%$ & $\mathrm{~N}$ & $\%$ & $\mathrm{n}$ & $\%$ \\
\hline Institucionalizada & & & & & & & & \\
Com alteração & 3 & 25 & 9 & 75 & 11 & 91,6 & 7 & 58,4 \\
Sem alteração & 9 & 75 & 3 & 25 & 1 & 8,4 & 5 & 41,6 \\
Total & 12 & 100 & 12 & 100 & 12 & 100 & 12 & 100 \\
\hline Não-institucionalizada & & & & & & & & \\
Com alteração & 3 & 50 & 2 & 33,33 & 3 & 50 & 1 & 16,6 \\
Sem alteração & 3 & 50 & 4 & 66,66 & 3 & 50 & 5 & 83,3 \\
Total & 6 & 100 & 6 & 100 & 6 & 100 & 6 & 100 \\
\hline Ambos os Grupos & & & & & & & & \\
Com alteração & 6 & 33,3 & 11 & 61,1 & 14 & 77,7 & 8 & 44,4 \\
Sem alteração & 12 & 66,6 & 7 & 38,8 & 4 & 22,2 & 10 & 55,5 \\
Total & 18 & 100 & 18 & 100 & 18 & 100 & 18 & 100 \\
\hline
\end{tabular}

Os motivos pelas quais as crianças do grupo 1 estavam institucionalizadas foram: $36,8 \%(\mathrm{n}=14)$ maus tratos, $13,2 \%(\mathrm{n}=5)$ abandono e $50 \%(\mathrm{n}=19)$ outros motivos, que poderiam ser a falta de condição sócio-econômica dos responsáveis ou a negligência dos mesmos. Dessas crianças $84,2 \%$ tem irmãos $(\mathrm{n}=32)$, sendo que $78,12 \%$ delas $(\mathrm{n}=25)$ vivem junto com seus irmãos.

Das crianças institucionalizadas, $81,6 \%$ $(\mathrm{n}=31)$ recebem visita dos pais, e todas freqüentam escola pública, 18,4\% $(n=7)$ em meio período e 81,6\% (n=31) em período integral. Nas instituições a idade das crianças abrigadas era de 0 a 18 anos, sendo que a convivência das crianças que participaram deste estudo, quando não estavam na escola, era de crianças da mesma idade $60,5 \%(\mathrm{n}=23)$ e de todas as idades $39,5 \%(n=15)$.

Devido à falta de informações, muitas questões não foram respondidas em relação às crianças institucionalizadas. As variáveis as quais não foi possível obtiver respostas foram: idade da mãe, realização de pré-natal, idade 
gestacional, peso ao nascimento, problemas durante a gravidez, tempo de amamentação. Dessa forma, não foi possível realizar a análise comparativa entre os dois grupos do estudo.

As seguintes variáveis que não mostraram associação significativa com suspeita de atraso no DNPM na análise bivariada foram: sexo, irmão mais velho mora junto, faixa etária das crianças que convive, visita dos pais, problemas de saúde (Tabela 4).

Ao realizar-se o teste $t$, verificou-se que o tempo de institucionalização e a quantidade de funcionários responsáveis não tiveram influência sobre o atraso no DNPM das crianças institucionalizadas ( $\mathrm{p}=0,584$ e $\mathrm{p}=0,182$ res- pectivamente). Os funcionários responsáveis pelas crianças institucionalizadas tinham como qualificação: $65,8 \%$ educadores e $34,2 \%$ voluntários.

\section{DISCUSSÃO}

A partir dos resultados, pode-se afirmar que as crianças institucionalizadas que participaram deste estudo não apresentaram suspeita de atraso DNPM em relação às crianças não institucionalizadas, ainda que a prevalência de suspeita de atraso fosse maior entre as crianças institucionalizadas.

Tabela 4: Teste de Denver II aplicado nos pré escolares de abrigos.

\begin{tabular}{|c|c|c|c|c|}
\hline \multirow[b]{3}{*}{ Variáveis } & \multicolumn{3}{|c|}{ Desenvolvimento Neuropsicomotor } & \multirow[b]{3}{*}{$\mathbf{P}$} \\
\hline & \multicolumn{2}{|c|}{ Suspeita de atraso } & \multirow[b]{2}{*}{ Total } & \\
\hline & n $(\%)$ & n (\%) & & \\
\hline \multicolumn{4}{|l|}{ Motivo do abrigo } & \\
\hline Maus tratos & $5(13,16)$ & $9(23,68)$ & $14(36,84)$ & 0,81 \\
\hline Abandono & $1(2,63)$ & $4(10,53)$ & $5(13,16)$ & \\
\hline Outros & $6(15,79)$ & $13(34,21)$ & $19(50)$ & \\
\hline Total & $12(31,58)$ & $26(68,42)$ & $38(100)$ & \\
\hline \multicolumn{5}{|l|}{ Sexo da criança } \\
\hline Feminino & $7(18,42)$ & $11(28,95)$ & $18(47,37)$ & 0,49 \\
\hline Masculino & $5(13,16)$ & $15(39,47)$ & $20(52,63)$ & \\
\hline Total & $12(31,58)$ & $26(68,42)$ & $38(100)$ & \\
\hline \multicolumn{5}{|c|}{ Idade das crianças que brinca } \\
\hline Mesma idade & $10(26,32)$ & $13(34,21)$ & $23(60,53)$ & 0,08 \\
\hline Todas as idades ( 0 a 18 anos) & $2(5,26)$ & $13(34,21)$ & $15(39,47)$ & \\
\hline Total & $12(31,58)$ & $26(68,42)$ & $38(100)$ & \\
\hline \multicolumn{5}{|c|}{ Criança recebe visita dos pais } \\
\hline Sim & $10(26,3)$ & $21(55,26)$ & 31() & 0,49 \\
\hline Não recebe visitas & $2(5,26)$ & $5(13,16)$ & $7(18,42)$ & \\
\hline Total & $12(31,56)$ & $26(68,42)$ & $38(100)$ & \\
\hline \multicolumn{5}{|c|}{ Mora com irmão mais velho } \\
\hline Sim & $10(26,32)$ & $20(52,63)$ & $30(78,95)$ & 0,61 \\
\hline Não & $2(5,26)$ & $6(15,79)$ & $8(21,05)$ & \\
\hline Total & $12(31,58)$ & $26(68,42)$ & $38(100)$ & \\
\hline
\end{tabular}


As crianças após começarem a freqüentar creches houve melhora nas áreas pessoalsocial, motora fina e motora grossa ${ }^{12}$. Observou-se neste estudo menor prevalência de suspeita de atraso nestas áreas, na qual todas as crianças institucionalizadas freqüentavam creche, em período integral ou meio período. Rezende e colaboradores ${ }^{12}$ relataram que não houve melhora na área da linguagem, assim como no presente estudo houve maior prevalência de suspeita de atraso nesta área. Para explicar esta prevalência, sugere-se a falta de interação dos responsáveis com a criança, que pode ser pela menor proporção de educador por crianças $^{12}$, pois neste estudo a proporção foi de aproximadamente 2 funcionários responsáveis por 10 crianças.

A relação estabelecida das crianças abrigadas com os responsáveis parece desempenhar um papel central na vida das crianças institucionalizadas, à medida que serão estes adultos que assumirão o papel de orientá-los e protegê-los ${ }^{7}$. Barros e Júnior ${ }^{13}$ descreveram em seu estudo etnográfico que houve falta de preparo das monitoras para cuidar das crianças institucionalizadas. No presente estudo, verificou-se que a maioria dos cuidadores tinha como qualificação educador $(65,8 \%)$, o que sugere que eles têm responsabilidade para propiciar um DNPM normal, porém não foi possível coletar dados suficientes para sustentar essa afirmativa.

O motivo de institucionalização e a visita dos pais podem influenciar o DNPM nas crianças institucionalizadas. O motivo de maior prevalência foi de maus-tratos $(36,8 \%)$, o que confere com o achado de Castanho e BlascoviAssis ${ }^{8}$, na qual em seu estudo mostrou que abandono e maus-tratos foram os motivos de maior incidência ( $43 \%$ e $37 \%$ respectivamente). Eles também relataram que $46 \%$ das crianças não recebem visitas. Porém no presente estudo verificou-se que, após a institucionalização a maior parte das crianças $(81,5 \%)$ recebe visita 1 ou 2 vezes por semana. Estes mes- mos autores ${ }^{8}$ ao avaliar o desenvolvimento motor de crianças institucionalizadas entre zero e dezoito meses, não observaram relação de atrasos motores com os fatores motivo ou tempo de abrigo e freqüência de visitas, dados semelhantes aos obtidos neste estudo.

Os questionários das crianças do abrigo não foram aplicados aos pais, muitas não tinham contato com os pais biológicos, portanto não foi possível obter as informações sobre idade gestacional, peso ao nascimento e tempo de amamentação. Essas variáveis, entre outras, são importantes fatores de risco para determinar suspeita de atraso ${ }^{14-17}$. Dessa forma, devido à falta de informações, não se pode afirmar que não ter a visita dos pais influenciou diretamente em uma suspeita de atraso significante no DNPM.

A instituição de abrigo se constitui uma fonte de apoio social mais próxima e organizada para as crianças, desempenhando um papel fundamental para o seu desenvolvimento ${ }^{7}$. Este ambiente institucional é carente de estímulos, ficando a criança mais jovem muitas vezes confinada no berço, limitando-a a explorar o ambiente, prejudicando seu desenvolvimento motor ${ }^{8}$. Outro fator que influencia no desenvolvimento motor são as práticas maternas utilizadas no cuidado diário de lactentes saudáveis ${ }^{18}$.

A privação materna que as crianças institucionalizadas possuem e uma provável falta de estímulo adequado dos funcionários permitem ter como hipótese que essas crianças teriam atraso em relação àquelas não institucionalizadas, porém neste estudo não houve atraso significante no DNPM. Esse achado confere com o estudo de Campos e Pereira ${ }^{19}$ que comparou o desenvolvimento das principais aquisições do primeiro ano de vida em crianças institucionalizadas e não institucionalizadas, verificando que em ambos os grupos existiram diferenças, porém não foram significativos. Por outro lado, Castanho e Blascovi-Assis ${ }^{8}$ encontraram que $97 \%$ dos participantes de sua pesquisa realizada com crianças institucionaliza- 
das de 0 a 18 meses tinham indicativo de atraso no DNPM, e esse indício teria como relação direta o ambiente e a falta de estimulação.

A variável sexo não teve relação com suspeita de atraso no DNPM, outros autores $^{20,21}$ também encontraram o mesmo achado em seus estudos.

Neste estudo, a não suspeita de atraso nas crianças institucionalizadas poderia ser explicada pela freqüência que essas crianças têm em escola pública, a maioria por período integral $(81,6 \%)$, e pela convivência das crianças abrigadas com uma quantidade maior de crianças, tanto da mesma idade quanto mais velha (0 a 18 anos). Verificou-se que foi significativo brincar com todas as idades e ter menos suspeita de atraso no DNPM ( $\mathrm{p}=0,077)$.

Ao conviver com crianças e adolescentes de diversas idades, as crianças institucionalizadas podem se envolver em parcerias uns com os outros, compartilhar sentimentos positivos e negativos, apoiando-se mutuamente ${ }^{7}$. O suporte familiar estabelece na vida da criança suas primeiras e mais importantes relações, obtendo experiências que serão a base para suas relações futuras ${ }^{22}$. Dessa forma, as crianças devem aprender brincando com as famílias e com outras crianças pelo relacionamento afetivo que os mesmos oferecem e esses estímulos são importantes no desenvolvimento intelectual, motor e emocional infantil, desde que sejam dados com afeto e sem ansiedade. A criança institucionalizada está privada desses estímulos, porém outro fator que poderia explicar o não atraso no desenvolvimento encontrado neste estudo é o apego entre as próprias crianças.

Entre as crianças institucionalizadas a interação que predomina é a brincadeira, sendo que essas crianças dispõem de longos períodos para recreação livre e tem mais oportunidades para se desenvolver em brincadeiras. Nessas brincadeiras que desenvolvem a relação de apego, pois após a separação de suas famílias elas tentam encontrar outras figuras de apego em diferentes situações ${ }^{23}$. Este fato poderia explicar como o suporte familiar pode ser suplantado pelo apego desenvolvido por essas crianças entre elas mesmas.

Vários autores citam a importância da nutrição ${ }^{19} \mathrm{e}$ de um ambiente adequado para um bom DNPM ${ }^{13-16,24-26}$. O brinquedo e os jogos influenciam no desenvolvimento das habilidades motoras da criança, e nas famílias com baixo nível socioeconômico, os estímulos são ineficientes para promover este desenvolvimento existe uma possibilidade de profissionais de áreas relacionadas ao movimento ajudem a estimular o desenvolvimento e de investimentos na criação de creches e espaços públicos, que atendam a essas necessidades ${ }^{27}$.

Nas etapas do desenvolvimento, todo aprendizado é influenciado por uma experiência previa, o individuo assimila uma informação á outras já existentes, e esta nova informação é adaptada a sua vida ${ }^{28}$.

De acordo com este estudo, o ambiente físico e a alimentação da instituição de abrigo estão adequados, parecendo não influenciar no desenvolvimento neuropsicomotor da criança institucionalizada.

Observou-se que os estímulos dos cuidadores associados às condições ambientais são de extrema importância para um DNPM pleno, embora nas instituições de abrigo houvesse uma privação do estímulo materno, podese sugerir que o suporte e apego familiar são suplantados pelo apego que as crianças adquirem ao conviver diariamente com um maior número de crianças.

Houve maior prevalência de suspeita de atraso no DNPM em crianças institucionalizadas, porém não foi estatisticamente significante ao se comparar com as crianças não institucionalizadas. Assim pode-se sugerir que uma intervenção seja realizada no ambiente institucional, tanto nos abrigos quanto nas escolas particulares, considerando-se principalmente a área da linguagem, por meio de uma equipe multidisciplinar e interdisciplinar, a fim de prevenir que ocorra o atraso no DNPM. 


\section{REFERÊNCIAS}

1. Bobath K. Uma base neurofisiológica para tratamento da paralisia cerebral. São Paulo: Manole; 1989. p. 1-2.

2. Tecklin JS. Fisioterapia Pediátrica. Porto Alegre: Artmed; 2002. p. 13-7.

3. Goto MMF, Gonçalves VMG, Netto AA. Classificação de recém-nascido e implicações clínicas no desenvolvimento neurológico: aspectos relacionados ao peso ao nascimento. Temas sobre desenvolvimento. $2004 ; 73$ (13): 26-34.

4. Landre FT, Bobbio TG, Gonçalves VMG, Barros Filho AA. Escalas de Avaliação Neuromotora em pré-escolares: revisão bibliográfica. Temas sobre desenvolvimento. 2005; 78(13): 46-52.

5. Shumway-cook, A, Woollacott M. Controle motor: teoria e aplicações práticas. Barueri, SP: Manole; 2003. p. 19-20.

6. Caetano MJD, Alves CR. Desenvolvimento motor de pré-escolares no intervalo de 13 meses. Rev Bras Cineantropom Desempenho Hum. 2005; 7(2): 05-13.

7. Siqueira, AC, Dell'aglio DD. O impacto da institucionalização na infância e na adolescência: uma revisão de literatura. Psicol Soc. 2006; 18(1): 71-80.

8. Castanho AAG, Blascovi-assis M. Caracterização do desenvolvimento motor da criança institucionalizada. Fisioterapia Brasil. 2004; 5(6): 437-42.

9. Gangliardo HGRG, Gabbard C, Gonçalves VMG. Coordenação visuomotora em lactentes de baixo peso ao nascimento: uma revisão de literatura. Temas sobre Desenvolvimento. 2002; 62(11): 51-5.

10. Filipini R. Crescimento e Desenvolvimento de Crianças Residentes em uma Favela do Município de São Paulo [dissertação]. São Paulo: Faculdade de Saúde Pública da USP; 2000 ; 21-38.

11. Frankenburg WK, Dodds J, Archer P, Bresnick B, Maschka P, Edelman N et al.
Denver II: Technical manual and training manual. Denver: Denver Developmental Materials, 1992.

12. Rezende MA, Beteli VC, Santos JLF. Follow-up of the child's motor abilities in day-care centers and pre-schools. Rev Latino-am. Enfermagem. 2005; 13(5): 619-25.

13. Barros RC, Junior GAF. Interações afetivas de crianças abrigadas: um estudo etnográfico. Ciênc \& Saúde Coletiva. 2007; 12(5): 1267-1276.

14. Andraca I, Pino P, La Parra A, Rivera F, Castillo M. Factores de riesgo para el desarrollo psicomotor em lactantes nascidos em óptimas condiciones biológicas. Rev Saúde Publica. 1998; 32(2): 138-47.

15. Halpern R, Giugliani ERJ, Victora CG, Barros FC, Horta BL. Fatores de Risco para suspeita de atraso no desenvolvimento neuropsicomotor aos 12 meses de vida. Rev Chil Pediatr. 2002; 73(5): 529-39.

16. Pilz EML, Schermann LB. Determinantes biológicos e ambientais no desenvolvimento neuropsicomotor em uma amostra de crianças de Canoas/RS. Ciênc Saúde Coletiva. 2007; 12(1): 181-90.

17. Kreling KCA, Brito ASJ, Matsu T. Fatores perinatais associados ao desenvolvimento neuropsicomotor de recém-nascidos de muito baixo peso. Pediatria. 2006; 28(2): 98-108.

18. Silva PL, Santos DCC, Gonçalves VMG. Influência de práticas maternas no desenvolvimento motor de lactentes do $6^{\circ}$ ao $12^{\circ}$ meses de vida. Rev bras fisioter. 2006; 10(2): 225-31.

19. Campos MCD, Pereira JS. Desenvolvimento neuromotor em crianças de 3 a 12 meses institucionalizadas e não institucionalizadas. Temas sobre Desenvolvimento. 2006; 15 (85-86): 57-61.

20. Rezende MA, Beteli VC, Santos JLF. Avaliação de habilidades de linguagem e pessoal-sociais pelo Teste de Denver II em instituições de educação infantil. Acta Paul Enferm. 2005; 18(1): 56-63. 
21. Passaglio KT, Neto RB, Andrade MC. Investigação teórico-prática do desenvolvimento motor de crianças de 2 a 11 anos. Neurociências. 2006; 3 (1).

22. Moura EW, Silva PAC. Fisioterapia: aspectos clínicos e práticos da reabilitação. São Paulo: Artes Médicas, 2005. p. 3-9.

23. Alexandre DT, Vieira ML. Relação de apego entre crianças institucionalizadas que vivem em situação de abrigo. Psicol Estud. 2004; 9(2): 207-17.

24. Caetano MJD, Silveira CRA. Desenvolvimento motor de pré-escolares no intervalo de 13 meses. Rev Bras Cineantropom Desempenho Hum. 2005; 7(2): 05-13.

25. Souto JN, Lima EAM, Paes JB, Bento MCC, Torquato JA, Saikai GPN,Silva A. Desenvolvimento neuropsicomotor de crianças institucionalizadas da zona leste da cidade de São Paulo. Temas sobre Desenvolvimento 2008;16(94):198-202.
26. Willrich A, Azevedo CCF, Fernandes JO.Desenvolvimento motor na infância: influencia dos fatores de risco e programas de intervenção. Ver Neurociencia 2009; 17(1):51-56.

27. Nobre FSS, Costa CLA, Oliveira DL, Cabral DA, Nobre GC, Caçola P. Análise das oportunidades para o desenvolvimento motor (affordances) em ambientes domésticos no Ceará - Brasil. Rev. bras. crescimento desenvolv. hum. 2009; 19 (1): 9-18.

28. Abreu LC, Oliveira MA, Carvalho TD et al. A epistemologia genética de Piaget e o construtivismo, Rev. Bras. Crescimento Desenvolv. Hum. 2010; 20(2): 361-366.

Recebido em: 08/jul./2009

Modificado em 02/jan./2010

Reapresentado em 29/nov./2010

Aceito em 28/mar./2011 